\title{
BMJ Open Multi-institution case-control and cohort study of risk factors for the development and mortality of Clostridium difficile infections in Japan
}

\author{
Masahiko Takahashi, ${ }^{1}$ Nobuaki Mori, ${ }^{2}$ Seiji Bito ${ }^{2}$
}

To cite: Takahashi M, Mori N, Bito S. Multiinstitution case-control and cohort study of risk factors for the development and mortality of Clostridium difficile infections in Japan. BMJ Open 2014:4:e005665. doi:10.1136/bmjopen-2014005665

- Prepublication history for this paper is available online. To view these files please visit the journal online (http://dx.doi.org/10.1136/ bmjopen-2014-005665).

Received 9 May 2014 Revised 5 August 2014 Accepted 8 August 2014

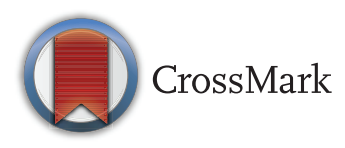

${ }^{1}$ Department of Gastroenterology, National Hospital Organization Tokyo Medical Center, Tokyo, Japan ${ }^{2}$ Department of General Internal Medicine, National Hospital Organization Tokyo Medical Center, Tokyo, Japan

Correspondence to Dr Masahiko Takahashi; matakaha@ntmc.hosp.go.jp

\section{ABSTRACT}

Objective: To examine risk factors for Clostridium difficile infection (CDI) morbidity and mortality in Japan.

Design: Multimethod investigation including a casecontrol study and cohort study.

Setting: 47 participating facilities of the National Hospital Organization (NHO).

Participants: 1026 patients with CDI and 878 patients in the control group over the age of 18 years admitted to the subject NHO facilities from November 2010 to October 2011.

Main outcome measures: In a case-control study, we identify risk factors for CDI development. Next, in a cohort study, we identify risk factors for all-cause mortality within 30 days following $C D I$ onset.

Results: A total of 1026 cases of CDI meeting the definitions of this investigation were identified, encompassing 878 patients at 42 of the 47 subject facilities. In the case-control study, we identified, compared with no antibiotics use, use of firstgeneration and second-generation cephem antibiotics (OR 1.44; $95 \% \mathrm{Cl} 1.10$ to 1.87 ), use of thirdgeneration and fourth-generation cephem antibiotics (OR 1.86; $95 \% \mathrm{Cl} 1.48$ to 2.33) and use of carbapenem antibiotics (OR 1.87; $95 \% \mathrm{Cl} 1.44$ to 2.42) the risk factors for $\mathrm{CDI}$ development. However, use of penicillin was not identified as a risk factor. In the cohort study, sufficient data for analysis was available for $924 \mathrm{CDI}$ cases; 102 of them (11.0\%) resulted in death within 30 days of $C D I$ onset. Compared with no anti-CDI drug use, use of vancomycin was associated with reduced risk of mortality (OR $0.43 ; 95 \% \mathrm{Cl} 0.25$ to 0.75 ) whereas metronidazole was not.

Conclusions: The findings mirror those of previous studies from Europe and North America, identifying the administration of broad-spectrum antibiotics as a risk factor for CDI development. The use of vancomycin is associated with a decreased risk of mortality.

\section{INTRODUCTION}

Clostridium difficile is the main causative pathogen of antibiotic-associated colitis. Since 2000, outbreaks of BI/NAP1/027

\section{Strengths and limitations of this study}

- This study is the first large-scale nationwide multicentre Clostridium difficile infection (CDI) investigation in Japan.

- Most of the epidemiological data of CDI have been limited to North America and Europe. Our data plays a role of completion of the missing data in Asia.

- Use of $\beta$-lactam antibiotics, except penicillin, was the risk factor for CDI development in the first Japanese large-scale investigation. Appropriate antibiotic use is necessary in order to control the incidence of CDI.

- Vancomycin administration for CDI was associated with decreased risk of mortality. Although the cost-effective treatment of CDI may necessitate the appropriate use of the less-expensive metronidazole, vancomycin should be administered in cases expected to become severe or life-threatening.

- The limitation of this study is the low number of registered CDI cases from quite a few participants and the existence of many confounding factors.

strain C. difficile infections (CDI) have been reported in North American and European hospitals and elder care facilities. The numbers of patients with CDI as well as severe and intractable cases have increased simultaneously. Consequently, epidemiological surveillance systems have been set up in several countries. However, very few countries have implemented such national-level measures.

CDI epidemiological studies in Japan to date have been based on scattered data from individual medical facilities. Consequently, the phenomenon of CDI in Japan is not sufficiently understood. ${ }^{1-9}$ Previous studies report that antibiotic administration is the largest risk factor for CDI development. Other risk factors include advanced age and proton pump inhibitor use. ${ }^{10}{ }^{11}$ CDI 
mortality rates differ depending on the presence or absence of an outbreak as well as the relevant definitions of epidemiological surveillance. Furthermore, it is especially difficult to objectively determine precise CDI-related mortality rates because of factors such as underlying patient conditions. ${ }^{12}$

This report documents a case-control study of CDI in Japan based on data from the National Hospital Organization (NHO), which is Japan's largest group of hospitals and includes facilities located nationwide. In addition, a cohort investigation of mortality among CDI cases was conducted.

\section{MATERIALS AND METHODS \\ Research design}

This multicenter study is a collaborative effort of the 47 facilities that met our facility standards from among the 143 NHO facilities in Japan. The study was planned as a part of the NHO's 'National Hospital Organization Multi-Center Clinical Research for Evidence-Based Medicine' project. This study was conducted with the approval of the Central Ethics Committee of the NHO. The CDI group in this study included, in principal, all newly diagnosed CDI cases among patients hospitalised from 1 November 2010 to 31 October 2011; cases were registered continuously.

In the case-control study of CDI development, CDI cases newly diagnosed during the investigation period were registered in the CDI group; meanwhile, agematched, sex-matched and underlying disease-matched patients in the same facilities were registered to the control group. In addition, a prospective cohort study of CDI group patients who died within 30 days of CDI development was conducted. This investigation is a multimethod study using standard case-control and cohort study designs.

\section{Definition of CDI}

CDI was defined as the presence of any gastrointestinal symptoms accompanied by a clinical suspicion of CDI as well as a positive result for $C$. difficile toxins from rapid stool testing or $C$. difficile isolation from stool cultures or both. Final determinations were carried out by the attending physician or the facility's infection control team.

Enzyme immunoassay testing kits for $C$. difficile toxins $\mathrm{A}$ and $\mathrm{B}$ were used as the rapid testing method (Immunocard CD toxin A\&B, Meridian Bioscience Inc, Cincinnati, Ohio, USA; C. Diff Quik Chek, Alere Medical Co., Ltd, Tokyo, Japan; Tox A/B Quik Chek, Nissui Pharmaceutical Co., Ltd, Tokyo, Japan; X/pect Toxin A/B, Kanto Chemical Co., Ltd, Tokyo, Japan). Cycloserine-cefoxitin mannitol agar (Nissuipure-to CCMA baichi EX, Nissui Pharmaceutical Co., Ltd, Tokyo, Japan), cycloserine-cefoxitin fructose agar (CCFA baichi, Becton, Dickinson and Company Co., Ltd, Tokyo, Japan; Poamedhia CCFA kairyoubaichi, Eiken Chemical Co., Ltd, Tokyo, Japan), and brucella HK agar
(RS) (brucella HK agar (RS), Kyokuto Pharmaceutical Industrial Co., Ltd, Tokyo, Japan) were used in the $C$. difficile isolation cultures.

\section{Case-control study of CDI development}

No additional information besides age, sex and date of diagnosis was gathered when new patients were registered in the CDI group. After the end of the study registration period, additional patient clinical data were gathered, including clinical department, underlying diseases, dates of hospital admittance and discharge and medical treatments administered for $\geq 3$ days between admittance and CDI development. Recorded medical treatments included disruption of feeding, parenteral nutrition, enteral feeding, surgery with general anaesthetics, cancer drugs, antibiotics (excluding external-use antibiotics) and proton pump inhibitors (oral or intravenous). We also collected data regarding the use of intravenous antibiotics including penicillins, firstgeneration and second-generation cephems, thirdgeneration and fourth-generation cephems, carbapenems, fluoroquinolones, clindamycin/lincomycin, anti-methicillin-resistant Staphylococcus aureus (MRSA) drugs and antifungal drugs, and others. Finally, we collected data regarding the use of oral antibiotics including cephems, fluoroquinolones and others.

The control group was divided into three subgroups according to age: $\leq 74,75-84$ and $\geq 85$ years. Control patients were selected from among patients at the same facilities who did not contract CDI and were matched to the patients with CDI with respect to age, sex, underlying disease and hospital stays of $\geq 5$ days within the same month as a counterpart's CDI diagnosis. The control group cases were selected regardless of gastrointestinal symptoms such as diarrhoea. We strove to ensure that the CDI and control groups were as matched as possible. The same data were collected from both groups. The control patients were registered and relevant patient data were gathered after the end of the CDI group study registration period.

\section{Cohort study on mortality among patients with CDI}

The prospective cohort study of registered CDI group patients from the case-control study examined all-cause mortality within 30 days as the primary outcome. If the registered patients were discharged within 30 days, clinical outcomes were not investigated after discharge in this study. The following data were collected: whether the underlying disease was infectious and whether comorbidities were related to malignant tumours (ie, gastrointestinal, respiratory, blood/lymph, gynaecological, urological, or other tumours including cancers of the ear, nose and throat), diabetes, renal failure, heart failure, respiratory failure or cirrhosis. In the cohort study, the definitions of severe complications were gastrointestinal perforations, toxic megacolon and CDI-related surgeries. Severe complications and the all-cause in-hospital mortality of patients within 30 days of CDI development were recorded. We 
also considered patient nutritional status including whether the patient was subjected to parenteral nutrition or enteral feeding as well as serum albumin levels measured within 30 days prior to CDI development (ie, $\geq 3.5$, 2.7-3.4 or $\leq 2.6 \mathrm{~g} / \mathrm{dL}$ ). In addition, we examined CDI treatment factors including whether antibiotic use was halted, probiotic use and the type of anti-CDI drugs used (ie, vancomycin and metronidazole). All patient data for the cohort investigation were collected after the end of the registration period.

\section{Data management and statistical analysis}

All input data were verified by a designated study data manager. Data from each facility were entered directly into a web-based case report form and subsequently encrypted for security. The data management centre was responsible for confirming any missing data and directly inquiring of the relevant facilities as necessary.

During the case-control phase of the study, CDI development was treated as the outcome and ORs were calculated from bivariate analysis comparing the use of different types of antibiotics as outcome causes. For each type of antibiotic, those used for $\geq 3$ days were designated 'used' while all others were designated 'unused.' A dummy variable regression was subsequently performed. Statistical significance in the bivariate analysis was tested by the $\chi^{2}$ test. Logistic regression analysis was performed using the individual patient characteristics and other assumed confounding variables as independent variables. The $95 \%$ CIs for each variable were used to determine the relationships between the various predictive variables and outcomes.

The clinical outcome of mortality within 30 days was set as the dependent variable and the relationships among the underlying diseases, nutritional status, probiotic use and types of anti-CDI drugs used were subjected to bivariate and multivariate analyses. Like the case-control phase, bivariate analysis was conducted using the $\chi^{2}$ test and the multivariate analysis was conducted using logistic regression. The significance level for all analyses was set at $p<0.05$. We used IBM SPSS Statistics V.20 for statistical analysis.

\section{Ethics committee approval and informed consent}

This study was conducted with the approval of the Central Ethics Committee of the NHO. In principle, individual patients who met the inclusion criteria were not given direct explanations of the study and no direct consent was sought. Information about the study was made public through postings on facility notice boards and webpages. Patients and their representative agents had the right to refuse study participation.

\section{RESULTS}

\section{Participating facilities}

Among the 47 facilities, a total of 1026 CDI cases were registered at 42 facilities throughout Japan, from
Hokkaido in the north to Okinawa in the south. No CDI cases were recorded at the remaining 5 participating facilities, more than 280 patient beds (table 1).

\section{Patient grouping}

A total of 1026 CDI cases that met the study definitions were recorded at the various institutions. We were unable to collect clinical records regarding medical treatments for 1 case; therefore, this case was excluded from the case-control study, and the remaining 1025 cases were analysed. A total of 962 patients (93.9\%) developed CDI within $48 \mathrm{~h}$ after hospital admittance. The control group comprised 878 patients who were selected from 41 of the 42 facilities. In the cohort study, we analysed the data from 924 of the 1025 CDI group patients, excluding 101 patients with no available recent serum albumin level data (ie, within 30 days prior to CDI development (figure 1).

\section{Case-control study of CDI development}

The mean ages of the CDI and control groups were 75.8 and 75.4 years, respectively. The majority of the participants were of advanced age: $64 \%$ and $62.5 \%$ of the CDI and control group patients were aged $\geq 75$ years, respectively. No significant differences were identified between the $\mathrm{CDI}$ and control groups in the univariate analysis of age distribution, sex differences or underlying disease (table 2). Among the medical treatments administered before CDI development, the following were significantly more prevalent in the CDI group than the control group: disruption of feeding (48.6\% vs $30.4 \%)$, parenteral nutrition (24.7\% vs $10.3 \%)$ and enteral feeding $(24.8 \%$ vs $9.1 \%)$. Antibiotics were used prior to CDI development in $85.8 \%$ of cases. The use of all types of intravenous antibiotics was significantly more prevalent in the CDI group. No significant differences were identified between the two groups with respect to oral antibiotic use. Meanwhile, in the univariate analysis, proton pump inhibitor use was significantly more prevalent in the CDI group than the control group (40.3\% vs $31.2 \%$ ).

We used logistic regression analysis to determine the risk factors for CDI development. The following medical treatments prior to CDI development were identified as significant risk factors in comparison to the control group: disruption of feeding (OR 1.31; 95\% CI 1.05 to 1.64 ), parenteral nutrition (OR 1.63; 95\% CI 1.21 to 2.20) and enteral feeding (OR 2.16; 95\% CI 1.60 to 2.92). The following intravenous antibiotics were also identified as statistically significant risk factors for CDI development: first-generation and second-generation cephems (OR 1.44; 95\% CI 1.10 to 1.87$)$, third-generation and fourth-generation cephems (OR 1.86; 95\% CI 1.48 to 2.33) and carbapenems (OR $1.87 ; 95 \%$ CI 1.44 to 2.42 ). However, penicillin (OR $1.04 ; 95 \%$ CI 0.82 to 1.33 ), fluoroquinolones (OR 1.16; $95 \%$ CI 0.74 to 1.83 ), 
Table 1 Number of registered cases of $\mathrm{CDI}$ and characteristics of hospitals included in the surveillance of CDI in the NHO (from November 2010 through October 2011)

\begin{tabular}{|c|c|c|c|c|c|c|c|}
\hline \multirow[b]{2}{*}{ Region } & \multirow[b]{2}{*}{$\begin{array}{l}\text { Number of } \\
\text { patient beds }\end{array}$} & \multirow[b]{2}{*}{$\begin{array}{l}\text { Number of } \\
\text { patient days }\end{array}$} & \multicolumn{2}{|c|}{$\begin{array}{l}\text { Number of patients } \\
\text { registered }\end{array}$} & \multirow[b]{2}{*}{$\begin{array}{l}30 \text {-day all-cause } \\
\text { mortality in } \\
\text { CDI group }\end{array}$} & \multicolumn{2}{|c|}{$\begin{array}{l}\text { Laboratory tests } \\
\text { used }\end{array}$} \\
\hline & & & $\begin{array}{l}\text { CDI } \\
\text { group }\end{array}$ & $\begin{array}{l}\text { Control } \\
\text { group }\end{array}$ & & $\begin{array}{l}\text { EIA for } \\
\text { toxins } \\
A \text { and B }\end{array}$ & Culture \\
\hline Hokkaido, & 698 & 208388 & 55 & 55 & $3(5 \%)$ & + & + \\
\hline \multirow[t]{4}{*}{ Tohoku } & 500 & 150603 & 42 & 32 & $1(2 \%)$ & + & + \\
\hline & 310 & 82687 & 28 & 19 & $2(7 \%)$ & & + \\
\hline & 310 & 72144 & 17 & 12 & $2(12 \%)$ & + & + \\
\hline & 220 & 76539 & 1 & 1 & $0(0 \%)$ & + & + \\
\hline Kanto, & 780 & 238420 & 124 & 121 & 15 (12\%) & + & + \\
\hline \multirow[t]{9}{*}{ Koshinetsu } & 455 & 151622 & 36 & 36 & $3(8 \%)$ & + & \\
\hline & 560 & 158921 & 35 & 30 & $4(11 \%)$ & + & + \\
\hline & 243 & 60155 & 34 & 34 & $6(18 \%)$ & + & + \\
\hline & 350 & 109025 & 22 & 22 & $4(18 \%)$ & + & + \\
\hline & 500 & 159432 & 15 & 14 & $1(7 \%)$ & + & \\
\hline & 510 & 166668 & 4 & 4 & $0(0 \%)$ & + & \\
\hline & 380 & 109482 & 3 & 2 & $0(0 \%)$ & + & + \\
\hline & 455 & 132483 & 3 & 1 & $0(0 \%)$ & + & \\
\hline & 429 & 104802 & 0 & 0 & $-(-)$ & + & \\
\hline Tokai, & 430 & 195209 & 42 & 26 & $10(24 \%)$ & + & + \\
\hline Hokuriku & 280 & 56475 & 0 & 0 & $-(-)$ & + & \\
\hline \multirow[t]{9}{*}{ Kinki } & 316 & 103677 & 24 & 22 & $1(4 \%)$ & + & \\
\hline & 220 & 47354 & 23 & 23 & $1(4 \%)$ & + & + \\
\hline & 600 & 191041 & 20 & 20 & $3(15 \%)$ & + & \\
\hline & 494 & 70455 & 15 & 15 & $6(40 \%)$ & + & + \\
\hline & 520 & 145299 & 13 & 9 & $1(8 \%)$ & + & \\
\hline & 500 & 142409 & 6 & 6 & $1(17 \%)$ & + & \\
\hline & 180 & 55721 & 3 & 3 & 1 (33\%) & + & \\
\hline & 346 & 118014 & 2 & 2 & $0(0 \%)$ & + & \\
\hline & 370 & 94722 & 0 & 0 & $-(-)$ & + & \\
\hline Chugoku, & 388 & 99728 & 54 & 49 & $5(9 \%)$ & + & + \\
\hline \multirow[t]{9}{*}{ Shikoku } & 700 & 211595 & 49 & 48 & $4(8 \%)$ & + & + \\
\hline & 506 & 119356 & 33 & 8 & $1(3 \%)$ & + & + \\
\hline & 400 & 122846 & 30 & 30 & $5(17 \%)$ & + & \\
\hline & 401 & 108303 & 26 & 0 & $2(8 \%)$ & + & + \\
\hline & 250 & 80558 & 21 & 21 & $0(0 \%)$ & + & \\
\hline & 424 & 128868 & 12 & 10 & $0(0 \%)$ & + & \\
\hline & 365 & 125645 & 10 & 10 & $3(30 \%)$ & + & + \\
\hline & 300 & 87061 & 0 & 0 & $-(-)$ & & + \\
\hline & 459 & 66454 & 0 & 0 & $-(-)$ & + & \\
\hline Kyushu, & 424 & 137827 & 46 & 22 & $5(11 \%)$ & + & \\
\hline \multirow{10}{*}{ Okinawa } & 702 & 239448 & 38 & 37 & $1(3 \%)$ & + & \\
\hline & 190 & 54038 & 33 & 31 & $9(27 \%)$ & + & \\
\hline & 550 & 189417 & 27 & 26 & $3(11 \%)$ & + & \\
\hline & 285 & 58185 & 25 & 25 & 3 (12\%) & + & \\
\hline & 500 & 140371 & 24 & 23 & $2(8 \%)$ & + & \\
\hline & 300 & 90457 & 14 & 14 & $4(29 \%)$ & + & \\
\hline & 320 & 103315 & 6 & 5 & $1(17 \%)$ & + & + \\
\hline & 280 & 79580 & 4 & 4 & $2(50 \%)$ & + & \\
\hline & 366 & 112906 & 4 & 4 & $0(0 \%)$ & + & \\
\hline & 368 & 89195 & 3 & 2 & $2(67 \%)$ & + & \\
\hline Total & 19486 & 5592077 & 1026 & 878 & $117(11 \%)$ & 45 & 20 \\
\hline
\end{tabular}

$\mathrm{CDI}$, Clostridium difficile infection; EIA, enzyme immunoassay; NHO, National Hospital Organization.

clindamycin/lincomycin (OR $1.35 ; 95 \%$ CI 0.81 to 2.26) and proton pump inhibitor use (OR 1.17; $95 \%$ CI 0.95 to 1.44$)$ were not identified as risk factors.
Cohort study on mortality among patients with CDI

The cohort study examined mortality among the 924 patients from the 1025 CDI group patients in the 


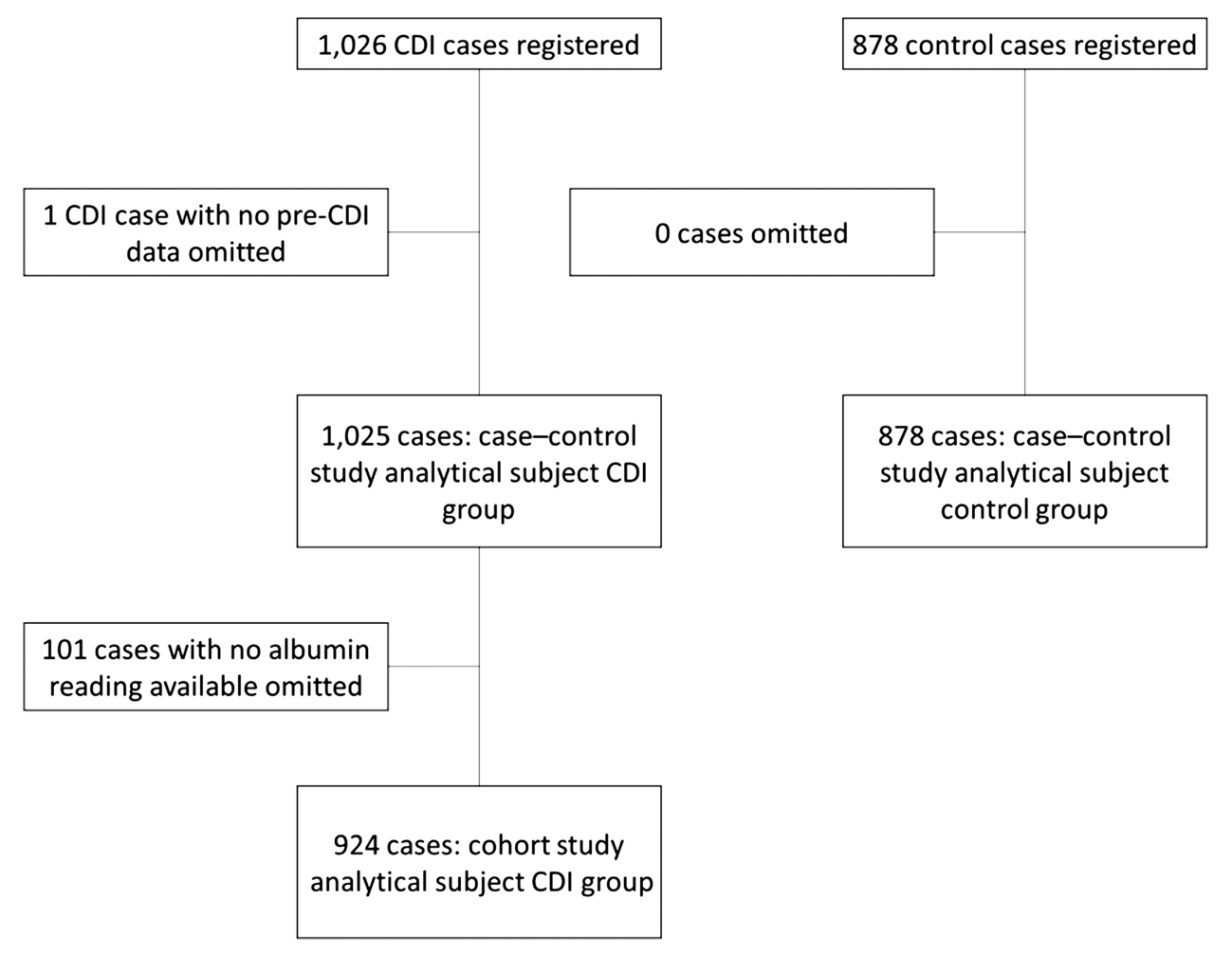

Figure 1 Study populations for the analysis of patients with Clostridium difficile infection (CDI) and controls.

case-control study for whom serum albumin level data before CDI development were available.

Among the 924 patients, $102(11.0 \%)$ died within 30 days of developing CDI. Among those cases, the cause of death was attributed to CDI in 11 cases $(1.2 \%)$. Of 11 patients, a patient had gastrointestinal perforation, another patient had CDI-related surgery and the others were not reported as severe complications. Toxic megacolon was reported in two patients, however, they had not died within 30 days of CDI development. The mean age of the 102 patients who died during the study was $80.1 \pm 8.3$ years. Patients $\geq 75$ years old were especially prevalent in this subgroup, accounting for $77.5 \%$ (79/ 102) of the cases.

Among the 714 cases in which CDI was treated directly, recurrence within 30 days was observed in 34 cases $(4.8 \%)$.

The univariate analysis indicated that comorbidities of heart and respiratory failure were significantly more prevalent among patients with CDI. In addition, lower serum albumin levels were significantly associated with mortality. Among CDI treatments, mortality was significantly lower among cases in which probiotics were administered.

A logistic regression analysis of the 102 cases in which the patients died within 30 days of CDI development was performed to identify the factors associated with the risk of mortality. Compared to patients $\leq 74$ years old, the OR of mortality among patients aged 75-84 years was 2.08 (95\% CI 1.19 to 3.62). Among underlying diseases, heart failure (OR 2.12; $95 \%$ CI 1.26 to 3.55) and respiratory failure (OR 1.98; 95\% CI 1.19 to 3.32) were identified as risk factors for mortality within 30 days of CDI development. Regarding nutritional status, neither parenteral nutrition nor enteral nutrition was identified as a risk factor for mortality. However, low serum albumin level (ie, $\leq 2.6 \mathrm{~g} / \mathrm{dL}$ ) was identified as a significant risk factor for mortality (OR 3.50; 95\% CI 1.33 to 9.22). Among CDI treatments, probiotic use (OR 0.66; $95 \%$ CI 0.42 to 1.04 ) was not identified as a risk factor for mortality. However, compared to cases in which no anti-CDI drugs were administered, vancomycin administration yielded an OR of 0.43 (95\% CI 0.25 to 0.75 ), indicating a significantly lowered risk of mortality in the CDI group. Meanwhile, no such lowered mortality was observed in cases treated with metronidazole (OR 0.85; $95 \%$ CI 0.48 to 1.51 ; table 3 ).

\section{DISCUSSION}

This is the first large-scale clinical study of CDI in Japan. This study examined 1026 cases of CDI recorded over 1 year at the nationwide facilities of Japan's largest hospital group. The findings of this investigation are similar to those reported in previous studies conducted in Europe, North America and Australia with respect to the identification of several risk factors for CDI development including age, severity of the underlying condition, artificial feeding and mortality. ${ }^{11} 13 \quad 14$ Antibiotic use is a known risk factor for CDI development. ${ }^{15}$ The present case-control study confirms that intravenous cephems and carbapenems are important risk factors. Some 
Table 2 Univariate and multivariate analyses of CDI development-related risk factors

\begin{tabular}{|c|c|c|c|c|c|}
\hline \multirow[b]{2}{*}{ Characteristics } & \multirow{2}{*}{$\begin{array}{l}\text { CDI group } \\
\text { Per cent }\end{array}$} & \multirow{2}{*}{$\begin{array}{l}\text { Control } \\
\text { group } \\
\text { Per cent }\end{array}$} & \multirow{2}{*}{$\begin{array}{l}\text { Univariate } \\
\text { analysis } \\
\text { p Value }\end{array}$} & \multicolumn{2}{|c|}{ Multivariate analysis } \\
\hline & & & & OR (95\% Cl) & p Value \\
\hline All & (1025) & (878) & - & - & - \\
\hline \multicolumn{6}{|l|}{ Age (years) } \\
\hline$\leq 74$ & $36.0(369)$ & 37.5 (329) & 0.67 & Ref. & - \\
\hline $75-84$ & $37.0(379)$ & $37.2(327)$ & & $1.02(0.81$ to 1.28$)$ & 0.88 \\
\hline$\geq 85$ & $27.0(277)$ & $25.3(222)$ & & $1.09(0.84$ to 1.41$)$ & 0.52 \\
\hline \multicolumn{6}{|l|}{ Sex } \\
\hline Women & $43.0(441)$ & $42.6(374)$ & 0.85 & $1.11(0.91$ to 1.36$)$ & 0.28 \\
\hline \multicolumn{6}{|l|}{ Underlying disease } \\
\hline Respiratory infections & $15.8(162)$ & $17.5(154)$ & 0.14 & - & - \\
\hline Other infectious conditions & $16.9(173)$ & $14.2(125)$ & & - & - \\
\hline Gastrointestinal conditions & 8.1 (83) & $9.0(79)$ & & - & - \\
\hline Malignant tumours & $22.6(232)$ & $24.3(213)$ & & - & - \\
\hline Cardiovascular conditions & $7.7(79)$ & $9.8(86)$ & & - & - \\
\hline Other conditions & $28.9(296)$ & $25.2(221)$ & & - & - \\
\hline \multicolumn{6}{|l|}{ Medical treatment prior to CDI development } \\
\hline Disruption of feeding & $48.6(498)$ & $30.4(267)$ & $<0.001$ & $1.31(1.05$ to 1.64$)$ & $<0.05$ \\
\hline Parenteral nutrition & 24.7 (253) & $10.3(90)$ & $<0.001$ & $1.63(1.21$ to 2.20$)$ & $<0.01$ \\
\hline Enteral feeding & $24.8(254)$ & $9.1(80)$ & $<0.001$ & $2.16(1.60$ to 2.92$)$ & $<0.001$ \\
\hline Surgery with general anaesthetic & $18.2(187)$ & $15.6(137)$ & 0.14 & $0.89(0.67$ to 1.18$)$ & 0.41 \\
\hline Cancer drugs & $11.3(116)$ & $14.2(125)$ & 0.06 & $0.86(0.62$ to 1.18$)$ & 0.35 \\
\hline Antibiotics use & $85.8(879)$ & $66.5(584)$ & $<0.001$ & - & - \\
\hline \multicolumn{6}{|l|}{ Intravenous } \\
\hline Penicillins & $27.6(283)$ & $21.0(184)$ & $<0.01$ & $1.04(0.82$ to 1.33$)$ & 0.75 \\
\hline First-generation/second-generation cephems & $22.7(233)$ & $15.6(137)$ & $<0.001$ & 1.44 (1.10 to 1.87$)$ & $<0.01$ \\
\hline Third-generation/fourth-generation cephems & $35.2(361)$ & $19.9(175)$ & $<0.001$ & $1.86(1.48$ to 2.33$)$ & $<0.001$ \\
\hline Carbapenems & $31.8(326)$ & $15.0(132)$ & $<0.001$ & 1.87 (1.44 to 2.42$)$ & $<0.001$ \\
\hline Fluoroquinolones & $7.5(77)$ & $4.0(35)$ & $<0.01$ & $1.16(0.74$ to 1.83$)$ & 0.52 \\
\hline Clindamycin/lincomycin & $6.5(67)$ & $2.8(25)$ & $<0.001$ & $1.35(0.81$ to 2.26$)$ & 0.25 \\
\hline MRSA drugs & $10.7(110)$ & $4.3(38)$ & $<0.001$ & $1.10(0.71$ to 1.72$)$ & 0.66 \\
\hline Antifungal drugs & $6.9(71)$ & $3.2(28)$ & $<0.001$ & $1.01(0.60$ to 1.70$)$ & 0.96 \\
\hline Others (aminoglycosides, monobactam, etc) & $8.5(87)$ & $5.9(52)$ & $<0.05$ & 1.19 (0.80 to 1.77$)$ & 0.39 \\
\hline \multicolumn{6}{|l|}{ Oral } \\
\hline Cephems & $5.6(57)$ & $4.4(39)$ & 0.29 & 1.49 (0.95 to 2.32$)$ & 0.08 \\
\hline Fluoroquinolones & $14.5(149)$ & $11.5(101)$ & 0.06 & $1.11(0.82$ to 1.51$)$ & 0.49 \\
\hline Others (macrolides, penicillins, etc) & $14.0(144)$ & $13.9(122)$ & 0.95 & $0.84(0.63$ to 1.13$)$ & 0.26 \\
\hline Proton pump inhibitors & $40.3(413)$ & $31.2(274)$ & $<0.001$ & $1.17(0.95$ to 1.44$)$ & 0.14 \\
\hline
\end{tabular}

$\mathrm{CDI}$, Clostridium difficile infection; MRSA, methicillin-resistant Staphylococcus aureus.

studies report a low risk of CDI development owing to intravenous penicillin administration. ${ }^{16} 17$ Concordantly, penicillin use was not identified as a risk factor in the present study. The proton pump inhibitor use was discussed as a risk factor for CDI development in the previous studies. ${ }^{18}{ }^{20}$ In the present logistic regression analysis, it was not identified as a risk factor.

In this study, $11.0 \%$ of patients with CDI died within 30 days. In comparison, higher 30-day mortality rates have been reported in previous outbreaks: $24.8 \%$ in the ribotype 027 strain outbreak in Canada and $36.7 \%$ in an examination of a single intensive care unit in the USA. ${ }^{21}$

${ }^{22}$ However, reports of non-outbreak conditions indicate mortality rates of $13 \%$, similar to the findings of the present study. ${ }^{23}$ Some reports state that the CDI-associated mortality rate has increased 2.5-fold, possibly indicating that CDI cases are more severe and contribute more significantly to mortality than previously thought. $^{12} 23$ The mortality rate of patients with CDI is reported to increase with age. ${ }^{24}$ Concordantly, the present study also found a significantly elevated risk of death in patients $\geq 75$ years old.

The findings of this study indicate that the mortality risk of patients with CDI was not reduced as a result of metronidazole treatment but was reduced with vancomycin treatment, corroborating the existing recommendation. ${ }^{25}$ It is worth noting that metronidazole is less expensive than vancomycin, making it economically advantageous. A patient's condition must be carefully evaluated when selecting anti-CDI drugs. In particular, for patients in the present study who had conditions associated with a greater mortality risk, including advanced age (ie, $\geq 75$ years), heart or respiratory failure or malnutrition as determined by low serum albumin levels, the 
Table 3 Univariate and multivariate analyses of all-cause mortality in patients with Clostridium difficile infection (CDI)

\begin{tabular}{|c|c|c|c|c|}
\hline \multirow[b]{2}{*}{ Characteristics } & \multirow{2}{*}{$\begin{array}{l}\text { All-cause mortality rate } \\
\text { Per cent }\end{array}$} & \multirow{2}{*}{$\begin{array}{l}\text { Univariate analysis } \\
\mathrm{p} \text { Value }\end{array}$} & \multicolumn{2}{|c|}{ Multivariate analysis } \\
\hline & & & OR (95\% Cl) & p Value \\
\hline All & $11.0(102 / 924)$ & - & - & - \\
\hline \multicolumn{5}{|l|}{ Age (years) } \\
\hline$\leq 74$ & $7.1(23 / 326)$ & & Ref. & \\
\hline $75-84$ & $13.3(47 / 353)$ & $<0.05$ & 2.08 (1.19 to 3.62$)$ & $<0.05$ \\
\hline$\geq 85$ & $13.1(32 / 245)$ & & 1.86 (0.98 to 3.55$)$ & 0.06 \\
\hline \multicolumn{5}{|l|}{ Sex } \\
\hline Men & $12.2(64 / 524)$ & & Ref. & \\
\hline Women & $9.5(38 / 400)$ & 0.21 & $0.78(0.49$ to 1.24$)$ & 0.29 \\
\hline \multicolumn{5}{|l|}{ Underlying disease } \\
\hline Non-infectious & $10.3(64 / 619)$ & & Ref. & \\
\hline Infectious & $12.5(38 / 305)$ & 0.37 & 0.99 (0.60 to 1.62$)$ & 0.97 \\
\hline \multicolumn{5}{|l|}{ Comorbidities } \\
\hline \multicolumn{5}{|l|}{ Malignant tumours } \\
\hline Not present & $10.6(67 / 630)$ & & Ref. & \\
\hline Present & $11.9(35 / 294)$ & 0.57 & 1.54 (0.94 to 2.53$)$ & 0.09 \\
\hline \multicolumn{5}{|l|}{ Diabetes } \\
\hline Not present & $11.6(89 / 765)$ & & Ref. & \\
\hline Present & $8.2(13 / 159)$ & 0.27 & $0.71(0.37$ to 1.35$)$ & 0.29 \\
\hline \multicolumn{5}{|l|}{ Renal failure } \\
\hline Not present & $10.7(84 / 784)$ & & Ref. & \\
\hline Present & $12.9(18 / 140)$ & 0.46 & $0.90(0.49$ to 1.65$)$ & 0.73 \\
\hline \multicolumn{5}{|l|}{ Heart failure } \\
\hline Not present & $9.3(70 / 756)$ & & Ref. & \\
\hline Present & $19.0(32 / 168)$ & $<0.01$ & $2.12(1.26$ to 3.55$)$ & $<0.01$ \\
\hline \multicolumn{5}{|l|}{ Respiratory failure } \\
\hline Not present & $9.2(69 / 754)$ & & Ref. & \\
\hline Present & $19.4(33 / 170)$ & $<0.001$ & 1.98 (1.19 to 3.32$)$ & $<0.01$ \\
\hline \multicolumn{5}{|l|}{ Cirrhosis } \\
\hline Not present & $11.2(100 / 895)$ & & Ref. & \\
\hline Present & $6.9(2 / 29)$ & 0.76 & $0.61(0.13$ to 2.83$)$ & 0.53 \\
\hline \multicolumn{5}{|l|}{ Indicators of nutritional status } \\
\hline \multicolumn{5}{|l|}{ Parenteral nutrition or enteral feeding } \\
\hline Not present & $9.4(53 / 563)$ & & Ref. & \\
\hline Present & $13.6(49 / 361)$ & 0.05 & $1.16(0.73$ to 1.84$)$ & 0.53 \\
\hline \multicolumn{5}{|l|}{ Serum albumin ( $g / d L)$} \\
\hline$\geq 3.5$ & $4.0(5 / 124)$ & & Ref. & \\
\hline $2.7-3.4$ & $7.2(27 / 376)$ & $<0.001$ & 1.55 (0.57 to 4.21$)$ & 0.39 \\
\hline$\leq 2.6$ & $16.5(70 / 424)$ & & 3.50 (1.33 to 9.22$)$ & $<0.05$ \\
\hline \multicolumn{5}{|l|}{ CDI treatments } \\
\hline \multicolumn{5}{|l|}{ Cessation of antibiotics } \\
\hline Not present & $12.5(65 / 519)$ & & Ref. & \\
\hline Present & $9.1(37 / 405)$ & 0.11 & 0.77 (0.48 to 1.22$)$ & 0.26 \\
\hline \multicolumn{5}{|l|}{ Probiotics (for intestine treatment) } \\
\hline Not present & $13.8(52 / 378)$ & & Ref. & \\
\hline Present & $9.2(50 / 546)$ & $<0.05$ & 0.66 (0.42 to 1.04$)$ & 0.08 \\
\hline \multicolumn{5}{|l|}{ Anti-CDI drugs } \\
\hline Not present & $15.2(32 / 210)$ & $<0.05$ & Ref. & \\
\hline Vancomycin alone & $7.4(32 / 433)$ & & $0.43(0.25$ to 0.75$)$ & $<0.01$ \\
\hline Metronidazole alone & $13.5(32 / 237)$ & & $0.85(0.48$ to 1.51$)$ & 0.59 \\
\hline Vancomycin and metronidazole & $13.6(6 / 44)$ & & 0.75 (0.27 to 2.08$)$ & 0.57 \\
\hline
\end{tabular}

use of vancomycin was expected to reduce the mortality. The recurrence rate was low $(4.8 \%)$ in this study compared to the previous studies. ${ }^{11}{ }^{26}$ We did not investigate those patients who developed CDI after 30 days nor did we investigate those patients who were discharged, even if within 30 days of CDI development. Therefore, the recurrence rate might be underestimated.

Regardless, this study also has several methodological limitations. The most salient being the low number of registered CDI cases from quite a few participants. In 
the definition of CDI, the times of diarrhoea were not investigated. Another limitation of the case-control study phase is the existence of many confounding factors. In particular, probiotic use, which was recently discussed to be correlated with CDI prevention, was not included in the predictive model of this study. ${ }^{10} 1127$ When interpreting the findings of this study, it is necessary to consider the influence of confounding factors that were not included in the analytical models. Regarding antibiotic use, the present analyses included independent explanatory variables for each antibiotic. However, actual antibiotic use is more complicated. Therefore, it is difficult to clearly determine the roles of individual antibiotics as risk factors for CDI development. Concerning matching process, we tried to adopt 1 to 1 pair sampling matched with sex, age group and main diagnosis. Some hospitals could not find appropriate control samples well matched with case samples. So the total number of the control group was less than that of the case sample. In addition, although data for the control group were analysed during the entire study period until hospital discharge, only data from the period prior to CDI development were analysed in the CDI group. Therefore, the risks might be underestimated, because the control group had a longer period of exposure risk than the CDI group did. Confounding factors that were not included in the present analyses also represent a limitation of the cohort study phase. Furthermore, issues of data quality among the facilities affect all aspects of this study. More than 40 different facilities participated in this study. While some facilities registered nearly all of their patients with CDI, other facilities registered smaller proportions of patients. Only C. difficile culture but not toxin test was used for the laboratory test in two facilities. Finally, there might have been differences with regard to individual researchers' understanding of the outcome definitions.

In order to ensure appropriate antibiotic use and control the incidence of CDI, it is important to create institutional measures such as infection control teams. The cost-effective treatment of CDI may necessitate the appropriate use of less-expensive metronidazole. However, in cases expected to become severe or lifethreatening, the more expensive drug vancomycin should be administered. CDI is one of many issues concerning medicine and medical treatment costs. Accordingly, further and more proactive research into CDI epidemiology is needed.

Acknowledgements The authors would like to express their sincere gratitude to Dr Haru Kato and the Department of Bacteriology II, National Institute of Infectious Diseases, Tokyo, Japan for their expert advice regarding CDI and the provision of CDI training to the participating facilities. They also wish to thank the participating institutions in the CD-NHO Study Group for their collaboration with data and sample collection: Hisaji Oshima (NHO Tokyo Medical Center); Hiroshi Miki (NHO Sendai Medical Center); Keisei Shimoe (NHO Fukuyama Medical Center); Harumi Tominaga (NHO Kure Medical Center); Toyomitsu Sawai and Eisuke Sasaki (NHO Ureshino Medical Center); Shie Nishijima and Naoko Maeda (NHO Shizuoka Medical Center); Masaru Amishima (NHO Hokkaido Medical Center); Miki Odawara (NHO Kyushu
Medical Center); Mitsuhiro Kamimura (NHO National Disaster Medical Center); Hideaki Nagai (NHO Tokyo National Hospital); Kiyoshi Furuta (NHO Matsumoto Medical Center, Matsumoto Hospital); Tohru Yamanaka (NHO Kumamoto Minami Hospital); Ikuko Mizouchi (NHO Minimi-Okayama Medical Center); Yutaka Sato (NHO Kanmon Medical Center); Keita Ato and Hiroki Saito (NHO Asahikawa Medical Center); Yoshio Haga (NHO Kumamoto Medical Center); Isao Murakami (NHO Higashihiroshima Medical Center); Takeshi Yamaryo (NHO Nagasaki Kawatana Medical Center); Hiroyuki Akiyama and Yukino Yoshikura (NHO Minami Wakayama Medical Center); Akiko Muratake (NHO Beppu Medical Center); Masato Hasegawa (NHO Higashi-Ohm General Medical Center); Isamu Kamimaki (NHO Saitama National Hospital); Tomoaki Kosyoubu (NHO Yonago Medical Center); Takao Odagaki (NHO Kyoto Medical Center); Nozomu Iwashiro (NHO Hakodate National Hospital); Hiroyasu Ishida (NHO Mito Medical Center); Hiroshi Komatsu (NHO Maizuru Medical Center); Kaoru Nakama (NHO Oita Medical Center); Yoshiko Yamamoto (NHO Osaka Minami Medical Center); Yoshihito Iwahara (NHO Kochi National Hospital); Fumiko Okino (NHO Yamaguchi-Ube Medical Center); Daisuke Higuchi (NHO Okinawa National Hospital); Kazuhiro Satonaka (NHO Hyogo-Chuo National Hospital); Takayoshi Soga and Haruko Ideguchi (NHO Yokohama Medical Center); Mayuko Watanabe (NHO Kagoshima Medical Center); Kozaburo Hiramatsu (NHO Nagasaki National Hospital); Mitsugu Saito (NHO Awara National Hospital); Morio Sawamura (NHO Nishigunma National Hospital); Satoru Kaneda (NHO Chiba Medical Center); Kenji Okada (NHO Fukuoka National Hospital); Katsuhiro Suzuki (NHO Kinki-Chuo Chest Medical Center); Tetsuko Chiba and Keiji Chida (NHO Iwate National Hospital); Akihiko Tamura (NHO Tochigi Medical Center); Shunji Matsuda (NHO Ehime Medical Center); Takaya Maruyama (NHO Mie National Hospital); Shigeaki Kimura (NHO Tokushima National Hospital); Shin Oguri (NHO Minami Kyoto National Hospital).

Contributors MT conceived the idea for the study, designed the study, developed the protocol, was responsible for study management and data collection, interpreted the findings and drafted the paper. NM contributed to the data analysis and interpretation of the findings and drafted the paper. SB designed the study, developed the protocol, performed the data analysis and interpreted the findings, and drafted the paper. All authors have read and approved the final manuscript.

Funding This study was supported by a grant from the National Hospital Organization (multicenter clinical studies for evidence-based medicine).

\section{Competing interests None.}

Ethics approval The Central Ethics Committee of the NHO.

Provenance and peer review Not commissioned; externally peer reviewed.

Data sharing statement No additional data are available.

Open Access This is an Open Access article distributed in accordance with the Creative Commons Attribution Non Commercial (CC BY-NC 4.0) license, which permits others to distribute, remix, adapt, build upon this work noncommercially, and license their derivative works on different terms, provided the original work is properly cited and the use is non-commercial. See: http:// creativecommons.org/licenses/by-nc/4.0/

\section{REFERENCES}

1. Honda $H$, Yamazaki $A$, Sato $Y$, et al. Incidence and mortality associated with Clostridium difficile infection at a Japanese tertiary care center. Anaerobe 2014;25:5-10.

2. Kato $\mathrm{H}$, Ito $\mathrm{Y}$, van den Berg $\mathrm{RJ}$, et al. First isolation of Clostridium difficile 027 in Japan. Euro Surveill 2007;12:E070111.3.

3. Kato H, Kato N, Watanabe K, et al. Analysis of Clostridium difficile isolates from nosocomial outbreaks at three hospitals in diverse areas of Japan. J Clin Microbiol 2001;39:1391-5.

4. Tagashira $Y$, Kato $H$, Senoh $M$, et al. Two cases of fulminant colitis due to binary toxin-positive Clostridium difficile that are not PCR ribotype 027 or type 078. J Med Microbiol 2013;62:1486-9.

5. Sawabe E, Kato H, Osawa K, et al. Molecular analysis of Clostridium difficile at a university teaching hospital in Japan: a shift in the predominant type over a five-year period. Eur J Clin Microbiol Infect Dis 2007;26:695-703.

6. Iwashima $\mathrm{Y}$, Nakamura $\mathrm{A}$, Kato $\mathrm{H}$, et al. A retrospective study of the epidemiology of Clostridium difficile infection at a University Hospital 
in Japan: genotypic features of the isolates and clinical characteristics of the patients. $J$ Infect Chemother 2010;16:329-33.

7. Kato $\mathrm{H}$, Ito $\mathrm{Y}$, Akahane $\mathrm{T}$, et al. Typing of Clostridium difficile isolates endemic in Japan by sequencing of slpA and its application to direct typing. J Med Microbiol 2010;59:556-62.

8. Collins DA, Hawkey PM, Riley TV. Epidemiology of Clostridium difficile infection in Asia. Antimicrob Resist Infect Control 2013;2:21.

9. Nakamura I, Yamaguchi T, Tsukimori A, et al. Fulminant colitis from Clostridium difficile infection, the epidemic strain ribotype 027, in Japan. J Infect Chemother 2014;20:380-3.

10. Rupnik M, Wilcox MH, Gerding DN. Clostridium difficile infection: new developments in epidemiology and pathogenesis. Nat Rev Microbiol 2009;7:526-36.

11. Cohen SH, Gerding DN, Johnson S, et al. Clinical practice guidelines for Clostridium difficile infection in adults: 2010 update by the society for healthcare epidemiology of America (SHEA) and the infectious diseases society of America (IDSA). Infect Control Hosp Epidemiol 2010;31:431-55.

12. Mitchell BG, Gardner A. Mortality and Clostridium difficile infection: a review. Antimicrob Resist Infect Control 2012;1:20.

13. Freeman J, Bauer MP, Baines SD, et al. The changing epidemiology of Clostridium difficile infections. Clin Microbiol Rev 2010;23:529-49.

14. Bliss DZ, Johnson S, Savik K, et al. Acquisition of Clostridium difficile and Clostridium difficile-associated diarrhea in hospitalized patients receiving tube feeding. Ann Intern Med 1998;129:1012-19.

15. Owens RC Jr, Donskey CJ, Gaynes RP, et al. Antimicrobial-associated risk factors for Clostridium difficile infection. Clin Infect Dis 2008:46(Suppl 1):S19-31.

16. Brown KA, Khanafer N, Daneman N, et al. Meta-analysis of antibiotics and the risk of community-associated Clostridium difficile infection. Antimicrob Agents Chemother 2013;57:2326-32.

17. Deshpande A, Pasupuleti V, Thota $P$, et al. Community-associated Clostridium difficile infection and antibiotics: a meta-analysis. J Antimicrob Chemother 2013;68:1951-61.
18. Dial S, Delaney JA, Barkun AN, et al. Use of gastric acidsuppressive agents and the risk of community-acquired Clostridium difficile-associated disease. JAMA 2005;294:2989-95.

19. Kwok CS, Arthur AK, Anibueze $\mathrm{Cl}$, et al. Risk of Clostridium difficile infection with acid suppressing drugs and antibiotics: meta-analysis. Am J Gastroenterol 2012;107:1011-19.

20. Tleyjeh IM, Bin Abdulhak AA, Riaz M, et al. Association between proton pump inhibitor therapy and Clostridium difficile infection: a contemporary systematic review and meta-analysis. PLOS ONE 2012;7:e50836.

21. Loo VG, Poirier L, Miller MA, et al. A predominantly clonal multi-institutional outbreak of Clostridium difficile-associated diarrhea with high morbidity and mortality. N Engl J Med 2005;353:2442-9.

22. Kenneally C, Rosini JM, Skrupky LP, et al. Analysis of 30-day mortality for Clostridium difficile-associated disease in the ICU setting. Chest 2007;132:418-24.

23. Hensgens MP, Goorhuis A, Dekkers OM, et al. All-cause and disease-specific mortality in hospitalized patients with Clostridium difficile infection: a multicenter cohort study. Clin Infect Dis 2013;56:1108-16.

24. Bloomfield MG, Sherwin JC, Gkrania-Klotsas E. Risk factors for mortality in Clostridium difficile infection in the general hospital population: a systematic review. J Hosp Infect 2012;82:1-12.

25. Zar FA, Bakkanagari SR, Moorthi KM, et al. A comparison of vancomycin and metronidazole for the treatment of Clostridium difficile-associated diarrhea, stratified by disease severity. Clin Infect Dis 2007:45:302-7.

26. Otete $\mathrm{EH}$, Ahankari AS, Jones $\mathrm{H}$, et al. Parameters for the mathematical modelling of Clostridium difficile acquisition and transmission: a systematic review. PLOS ONE 2013;8:e84224.

27. Johnston BC, Ma SS, Goldenberg JZ, et al. Probiotics for the prevention of Clostridium difficile-associated diarrhea: a systematic review and meta-analysis. Ann Intern Med 2012;157:878-88. 